\title{
Wake topology of curved cylinders at low Reynolds numbers
}

A. Miliou, S.J. Sherwin*, J.M.R. Graham

Department of Aeronautics, Imperial College of Science, Technology and Medicine, Prince Consort Road, London, SW7 2BY

\begin{abstract}
A numerical investigation into the flow past curved cylinders at Reynolds numbers of 100 and 500 has been performed. Two different configurations of a quarter turn with the free-stream flow vector parallel to the plane of curvature have been simulated. The vortex shedding patterns and the wake topology are studied and the sectional forces at different locations of the span are presented. The three-dimensional simulations were computed with a spectral/ $h p$ element method and the computational results were compared with flow visualisation experiments undertaken in the towing tank facility of the Department of Aeronautics of Imperial College.
\end{abstract}

\section{Introduction}

As the need to develop deep-water reservoirs for the exploitation of hydrocarbons below the sea has been increasing, riser pipes are now required to operate in depths up to $2000 \mathrm{~m}$. Catenary risers, which are effectively curved riser pipes, are preferred over vertical risers for deep-water reservoirs as they can accommodate larger motions of the floating vessel. A catenary riser is subject to fluid dynamic loading as a combination of waves and current and may be in tangential contact with the seabed while part of its length lies near horizontal at the seabed being semi- or even fully- buried occasionally. Understanding the unsteady flow pattern developed behind a deformed catenary riser pipe is a problem that requires investigation in three dimensions. The empirical models in commercial use are applied on a two-dimensional sectional basis. A sectional approach that assumes two-dimensional flow for a curved pipe, for the cases where the free-stream velocity vector is not normal to the centreline of the body, would fail at the sections where the vortices are not shed normal to the axis of the body.

With the motivation stemming from the offshore industry, an investigation into the vortex shedding patterns of curved cylinders at low Reynolds numbers has been conducted. A generic shape of a curved riser pipe would be that of a curved cylinder which is certainly of interest as a bluff body type of flow. The computational investigations

${ }^{*}$ author for correspondence, s.sherwin@ic.ac.uk

(C) 2002 Kluwer Academic Publishers. Printed in the Netherlands.

iutam_miliou.tex; 9/12/2002; $11: 32 ;$ p.1 
described in the current work have focused on gaining a better understanding of the mechanics of the flow past curved cylinders and their wake structures.

\section{Computational Method}

Computations have been performed using $\mathcal{N} \varepsilon \boldsymbol{\kappa} \mathcal{T} \boldsymbol{\alpha} \boldsymbol{r}[1,5,7]$, a threedimensional incompressible Navier-Stokes solver, based on the spectral/ $h p$ element method. The spectral method is a high-order discretisation method where one can increase simultaneously the number of elements (h-refinement) and the order, $\mathrm{P}$, of the polynomial expansion in the elemental domains (p-refinement). For smooth solutions the spectral $/ h p$ element method can achieve exponential convergence in terms of the polynomial order, P. Hybrid conforming discretisations, with tetrahedral and prismatic elements, have been used in the computational domain. The nature of the problem studied necessitated spectral element discretisations in all three directions and not just the Fourier extension to the two-dimensional version of the code.

The algorithm for the incompressible Navier-Stokes solver is based on a high-order stiffly stable splitting scheme [5]. The non-linear terms are solved explicitly and the linear terms are solved implicitly. This high-order splitting scheme propagates the velocity and pressure fields at time $n$ over a time step $\Delta t$ in three substeps in order to determine the fields at $n+1$. The first step takes care of the non-linear terms, the second step becomes the Poisson equation for pressure after imposing the divergence-free conditon on the second intermediate velocity field, and the third step deals with the linear terms. Hence, a Helmholtz equation is obtained for each of the velocity fields and a Poisson equation for the pressure field.

The mesh generation for all the computational investigations was accomplished with the package Felis $a$ where a modified advancing layers method [3] is employed near the pipe wall regions and a method based on the advancing front technique [4] is employed for the rest of the domain. Hybrid meshes were constructed where the boundary layer mesh was composed of prismatic elements growing in the direction of normal to the pipe surface and the interior of the domain was filled with tetrahedra.

iutam_miliou.tex; 9/12/2002; 11:32; p.2 


\section{Results and Discussion: Quarter turn in uniform flow parallel to the plane of curvature}

We have considered a generic shape for a catenary riser as a quarter turn (with or without a straight cylinder extension at the ends). There are effectively two configurations when the free-stream velocity vector is in the plane of curvature. Figure 1 represents one configuration. The second configuration is shown in figure 7 . The emphasis of this investigation is to study the vortex shedding patterns past curved cylinders under uniform flow in the plane of curvature where the fully threedimensional computations can provide more insight than a sectional approach.

\subsection{REYNOLDS NUMBER 100}

\subsubsection{First configuration}

The first configuration considered is shown in figure 1 and consists of a quarter turn with an in-line extension of ten diameters. The quarter turn part of this structure had a non-dimensional radius of curvature of 12.5 (non-dimensionalised by the cross-section diameter of the cylinder). The mesh comprised of 10250 elements, out of which 1184 were the prismatic surface elements.

Figure 1 depicts a cut in the computational domain at a plane including the free-stream vector. The flow is in the negative $x$-direction, from left to right as shown. Boundary B includes an intersection with the curved cylinder and so a symmetry boundary condition was imposed. Boundary A corresponds to the outflow plane and boundaries $\mathrm{C}$ and $\mathrm{D}$ correspond to free-stream boundaries where the uniform velocity

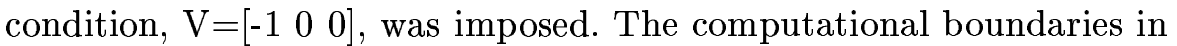
the $y$-direction were at a distance of 5 diameters from the cylinder axis and a uniform free-stream condition was also imposed on these planes.

The computations were initially performed using a second order polynomial and then restarted with fourth and sixth order polynomials. Using sixth order polynomials, the mesh accounted for 993,608 local degrees of freedom per variable. Blockage for these computational cases was $7.2 \%$.

Figure 2 depicts the force coefficients in the $x-, y$-, and $z$ - directions and the pressure contours at slices normal to the cylinder axis. The $x$-direction is the direction of the drag and the $y$ - and $z$-directions represent the horizontal and vertical component of the transverse force respectively. The transition from order 4 to order 6 occurs at time $t=$ 350.5. Moreover, one should note that the fluctuations about the mean and the range in the axes of the $x$ - and $z$-directions are significantly 


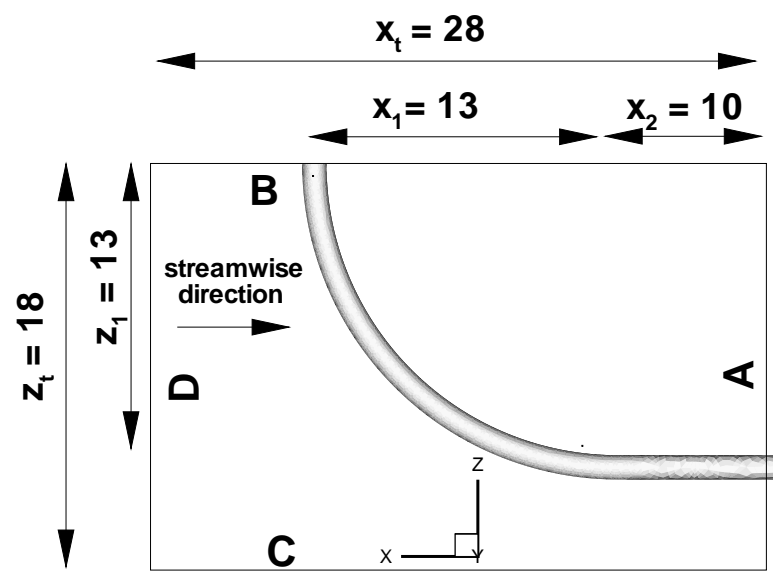

Figure 1. Computational boundaries (not to scale) for the first configuration

smaller compared to those in the $y$ - direction. Along the lower part of the curved cylinder as it becomes aligned with the free-stream, vortex shedding in the near wake becomes less vigorous. This is shown by the reduction of the pressure minimum in the sectional planes shown in figure 2 .
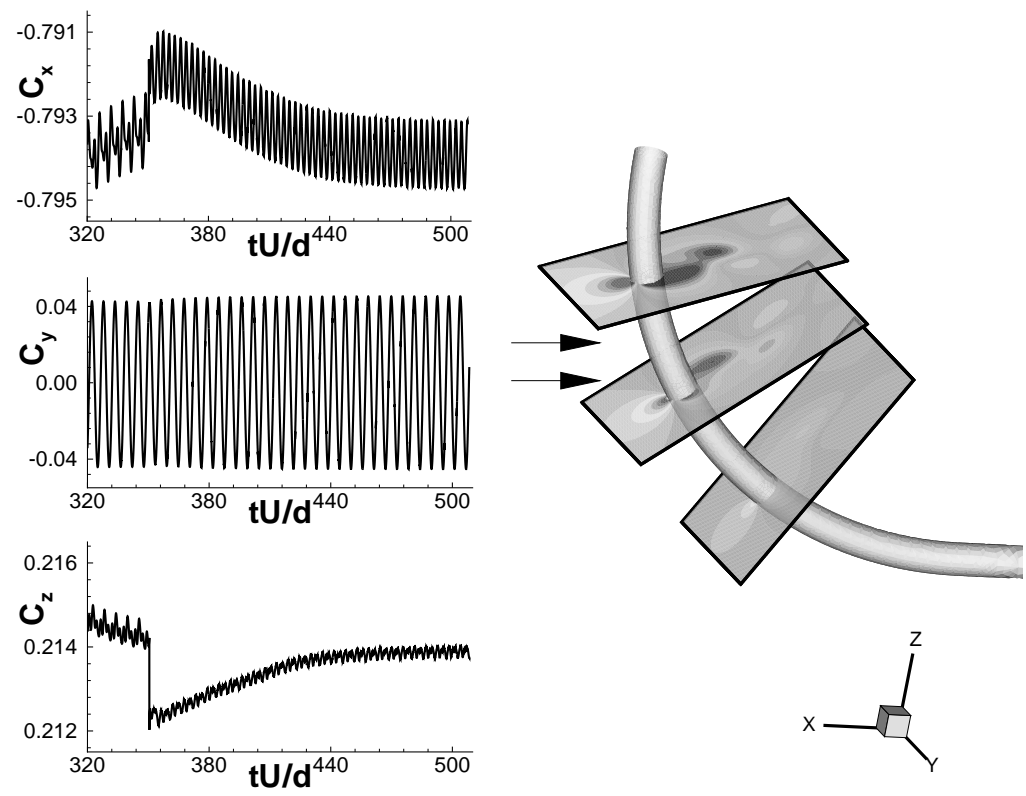

Figure 2. Force coefficients and pressure contours for a quarter turn with an in-line extension in uniform flow parallel to the plane of curvature, $R e=100$ 
As a general comment, the force coefficients produced for this case behave in a similar manner as those for the standard example of a straight two-dimensional cylinder. As figure 2 depicts, the deviations from the mean are much smaller for the drag force ( $x$-direction) than for the horizontal component ( $y$-direction) of the transverse force. The frequency of the drag was found to be equal to 0.35 and that of the horizontal component of the transverse force to be equal to 0.175 under the current blockage conditions; hence the similarity with the twodimensional cylinder which exhibits a drag force with a frequency equal to twice that of the lift force.

In order to investigate the wake structures developed, the $\lambda_{2}$ criterion presented by Jeong \& Hussain [2] was employed for identifying the vortex cores in the wake. The $\lambda_{2}$ criterion stems from the condition of flow rotation leading to a minimum of pressure in the absence of unsteady or viscous contributions. The second largest eigenvalue of $S^{2}+\Omega^{2}$ (where $S$ and $\Omega$ are the symmetric and anti-symmetric components of the velocity gradient tensor) must be negative in order to locate a vortex core.

As the 3D view in figure 3(a) illustrates, in the near wake the vortex cores are straight, with their axes normal to the free-stream, but start to distort further downstream in the wake. Figure 3(b) depicts a projection of the vortex cores on the $x y$-plane, i.e. view from the top, showing the anti-symmetric wake with a staggered array of vortices.

(a)

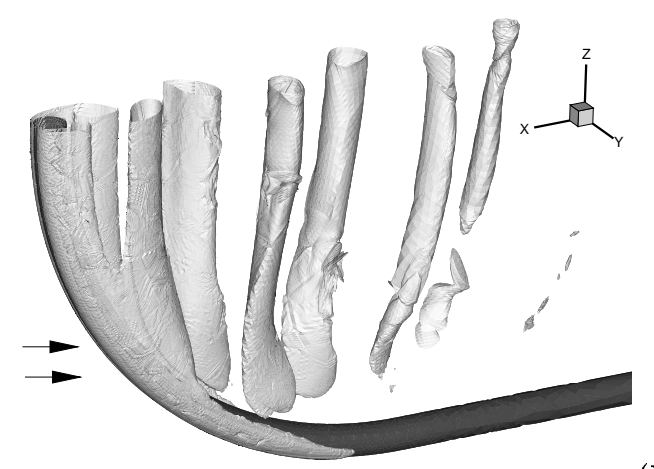

(b)

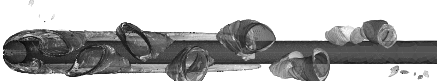

Figure 3. Vortex cores in the wake of a quarter turn with an in-line extension in uniform parallel flow, $R e=100, \lambda_{2}=-0.1$

Figure 4 shows the centreplane $(y=0)$ streamlines upstream of the curved cylinder. The streamlines show motion along the length of the curved cylinder towards the bottom of the geometry dictated by the shape of the stagnation face of the curved cylinder which tends to deflect the flow away from the curved cylinder. 


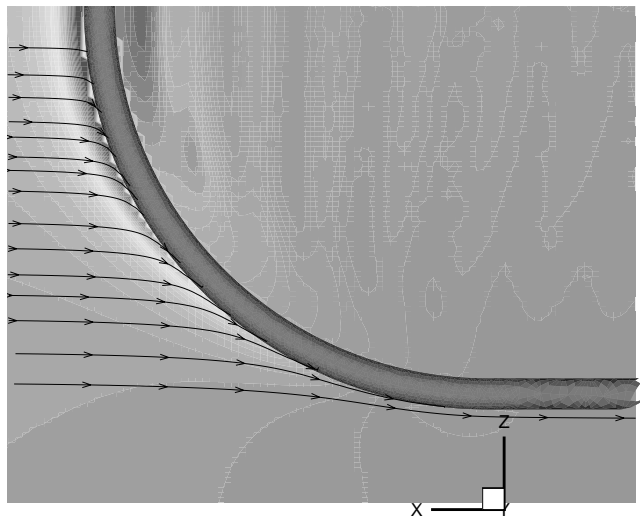

Figure 4. Centreplane streamlines $(y=0)$ for a quarter turn with an in-line extension in uniform parallel flow, $R e=100$

We note that even though the velocity profile at the inlet is uniform, there is an effective variation in the sectional local Reynolds number as the free-stream velocity vector is not normal to the centreline of the body at all sections. Therefore, the component of the incoming flow vector in the sectional planes varies over the sections. Following a twodimensional sectional argument there would be a limit at which the normal flow Reynolds number would have fallen below the threshold value for vortex shedding and vortices would not be shed.

The sectional forces for $C_{y}$ computed at slices normal to the cylinder axis at different locations of the span for this structure are depicted in figure 5. The top of the cylinder is at $S / d=0$ whereas $S / d=$ 19.6 corresponds to the vertical slice at the end of the quarter turn (S represents the arc length measured from the top). An arc length of $S / d=5$ corresponds to a section inclined approximately $23^{\circ}$ from the horizontal and a local Reynolds number of roughly 92 . Similarly, an arc length of $S / d=10$ corresponds to a section inclined about $46^{\circ}$ from the horizontal and a local Reynolds number of 69 whereas an arc length of $S / d=15$ corresponds to a section inclined about $69^{\circ}$ from the horizontal and a local Reynolds number of roughly 36 . This sectional local Reynolds number is lower than the critical Reynolds number of $47-49$ where the onset of vortex shedding for a circular straight cylinder occurs. A Reynolds number of 50 would correspond to an arc length of $S / d \approx 13$. Consistent with figure 3 , the strong alternating vortex shedding pattern at the top of the curved cylinder is also depicted in figure 5. A decrease in the magnitude of the forces can then be observed as we go down along the span (which shows the 
decreasing strength of vortices) and finally a phase change. The upper cylinder shedding drives the lower end.

(a)

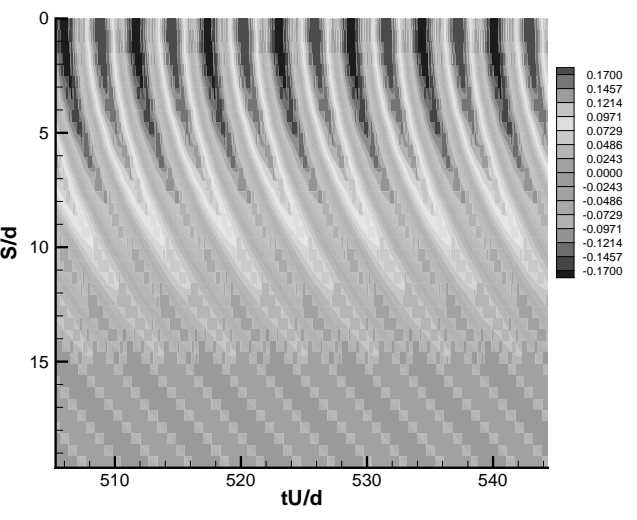

(b)

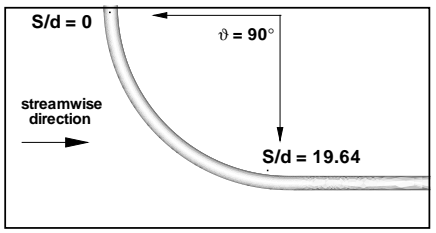

Figure 5. Sectional forces for $C_{y}$ along the axis of a quarter turn, $R e=100$

Experimental work was also conducted in order to compare the computational results with the flow visualisations. A model curved cylinder with the same aspect ratio as in the computational cases was towed in a tank [6] at an effective Reynolds number equal to that of the computations. The flow visualisation images obtained by illuminating dye diffused from the stagnation line of the curved cylinder have shown good qualitative agreement with the numerical investigations. The flow visualisation obtained for this case is shown in figure 6 and can be compared with figure 3. Note, that the slight bowing of the vortices towards the top cylinder end in experiments where a straight circular cylinder is towed through a water surface has been observed before and explained by Slaouti \& Gerrard [8]. Finally, the bending of the vortices further downstream in the wake is more apparent in the experiments than in the computations where the mesh is less refined far away from the curved cylinder.

\subsubsection{Second configuration}

The flow past the same quarter turn, but rotated by $180^{\circ}$ with respect to the $z$-axis was also computed. The non-dimensional radius of curvature for the quarter turn was again 12.5. The mesh comprised of 14847 elements, out of which 1574 were the prismatic surface elements. The computation was performed originally using a second order polynomial and was then restarted with fourth and sixth order polynomials. When the computation was performed using sixth order expansion, the mesh accounted for 1, 423, 436 local degrees of freedom per variable. Blockage for these computational cases was $3.9 \%$. 


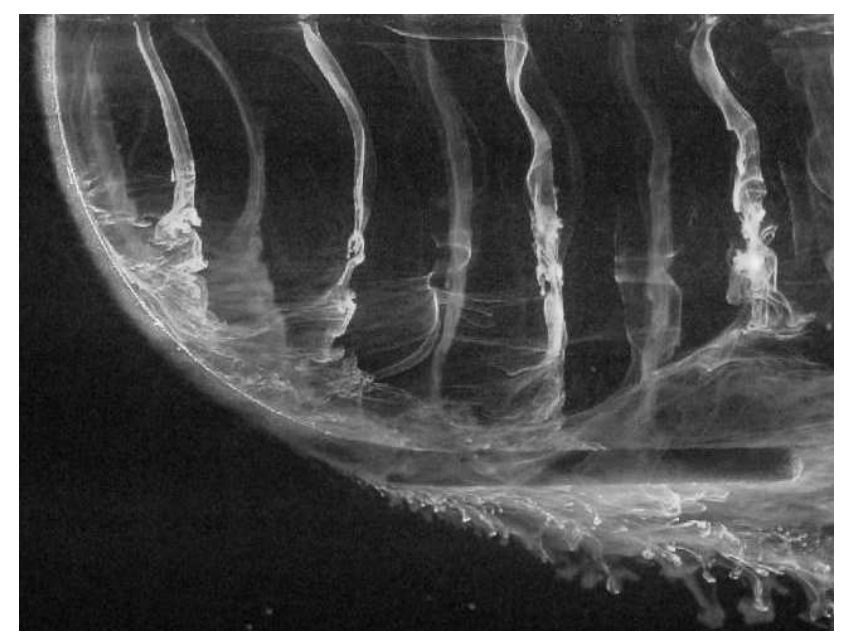

Figure 6. Flow visualisation for a quarter turn with an in-line extension in uniform flow parallel to the plane of curvature, $R e=100$

Figure 7 shows a cut in the computational domain at a plane including the free-stream vector. The flow is in the positive $x$-direction from left to right in the page. Boundary B includes an intersection with the curved pipe and so a symmetry boundary condition was imposed. Boundary D corresponds to the outflow plane and boundaries A and $\mathrm{C}$ correspond to free-stream boundaries where the uniform velocity condition, $\mathrm{V}=\left[\begin{array}{lll}1 & 0 & 0\end{array}\right]$ was imposed. The computational boundaries in the $y$-direction were at a distance of 8 diameters from the cylinder axis and a uniform free-stream condition was also imposed on these planes.

Contrary to case 3.1.1, this configuration, under the same uniform parallel to the plane of curvature flow, does not exhibit the vortex shedding features. As figure 8 shows, the forces are steady. Furthermore, the magnitude of the force coefficient in the $x$-direction is smaller by $12 \%$ compared to the previous case when both solutions have been approximated by fourth order polynomials.

Time $t=200$ in figure 8 represents the transition from a polynomial order of two to four and time $t=333$ represents the transition from a polynomial order of four to six. We note that there is a reduction of approximately $4 \%$ in the magnitude of $C_{x}$ as the order of the polynomial approximation is increased from two to four whereas a reduction of merely $0.1 \%$ as the polynomial order is increased from four to six.

Centreplane streamlines $(y=0)$ upstream of the curved cylinder are shown in figure 9 which can be directly compared with figure 4 . The streamlines still indicate motion along the length of the cylinder 


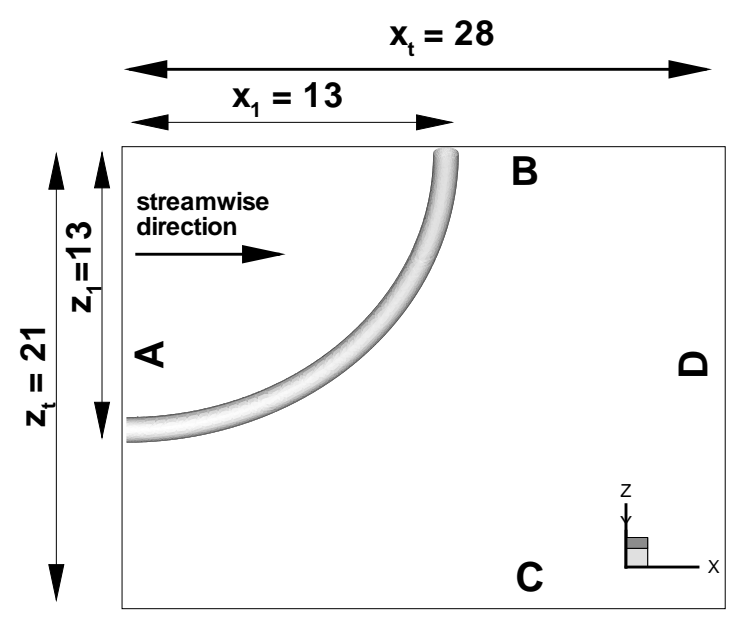

Figure 7. Computational boundaries (not to scale) for the second configuration
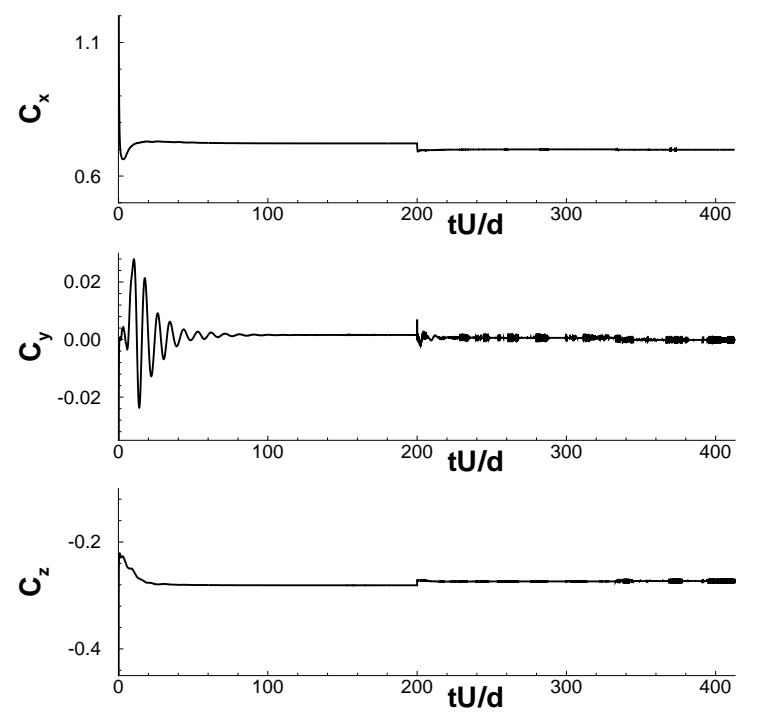

Figure 8. Force history for the second configuration, $R e=100$

but now is towards the top computational plane as the shape of the stagnation face now sets up the reverse flow mechanism.

Figure 9 also depicts the $u$-component velocity contours in the vicinity of the cylinder at four different horizontal planes. These velocity contours are consistent with the force plots indicating that periodic vortex shedding does not occur. Moreover, a variation in the wake 


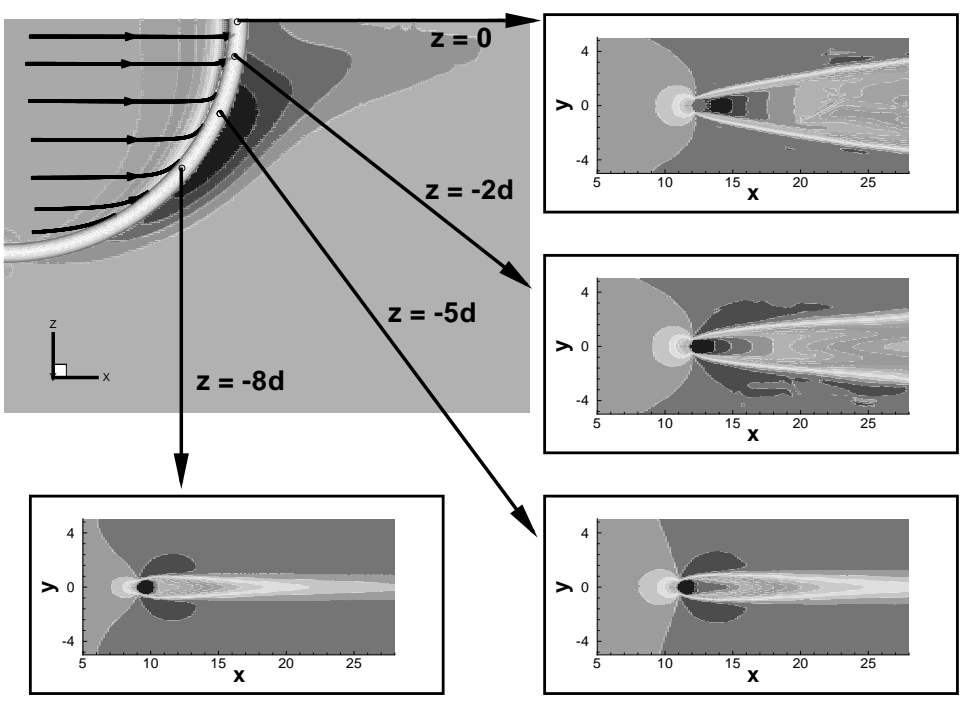

Figure 9. Centreplane streamlines $(y=0)$ and $u$-velocity contours at horizontal slices for a reverse quarter turn in uniform parallel flow, $R e=100$

width along the span can be observed from these slices of symmetric wakes with the top slice exhibiting the widest wake and the bottom one having the narrowest wake.

Similar qualitative results were obtained in the work of Darekar \& Sherwin [9] and Bearman \& Owen [10] on wavy cylinders. Leading edge cross-flow was found to be associated with streamwise vorticity which can result in the breakdown of the vortex street. Moreover, variation of the wake width was observed both in the computational and in the experimental work. The widest wake along the span of the wavy cylinder was found to be at the geometrical minimum, or "valley" defined as the most downstream cross-section [9]. In our case of the curved cylinder, the "geometrical minimum" in figure 9 is the top computational plane, $z=0$, which indeed exhibits the widest wake.

Furthermore, there are additional components of vorticity to the two-dimensional component. Figure 10(a) shows $w_{x}$, the horizontal component of vorticity in a vertical plane in the wake downstream of the cylinder. Variation of the strength of $w_{x}$ along this plane can also be observed. Plotting $w_{z}$, the spanwise vorticity in horizontal planes as shown in figure 10(b), we notice that the shear layers are not interacting and we observe again the variation in the wake width.

Finally, it should be noted that additional computations were performed in order to investigate the effect of blockage and of the top 

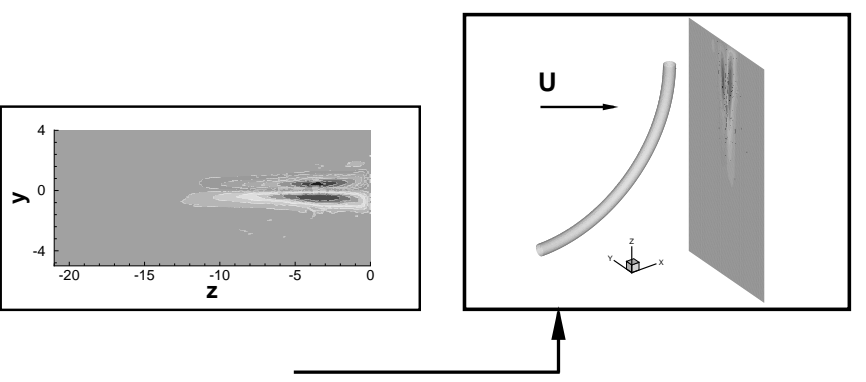

(a)

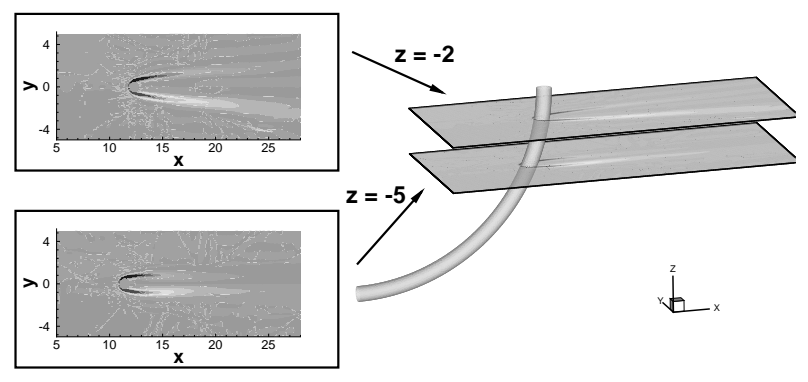

(b)

Figure 10. Streamwise vorticity on a vertical plane (a) and spanwise vorticity on a horizontal plane (b)

computational boundary being a symmetry plane (by adding a four diameter straight cylinder vertical extension on top) on the present results. These simulations have shown that the features presented here are qualitatively free from these effects.

\subsection{REYNOLDS NUMBER 500}

\subsubsection{First configuration}

The flow past the same quarter turn with a ten diameter horizontal extension studied in section 3.1.1 has also been computed at a higher Reynolds number, that of 500 to see the effects of introducing three dimensional instability in the wake, following the straight cylinder behaviour. Also the higher Reynolds number should tend to preserve shedding to a lower angle at the bottom. The computation was restarted from the flow field of case 3.1.1 at a Reynolds number of 100 using a fourth order polynomial expansion.

Figure 11 shows the integrated force coefficients in the $x-, y-$, and $z$ - directions. The $x$ - direction is the direction of the drag and the $y$ - and $z$-directions represent the horizontal and vertical component of the transverse force respectively. Time $t \approx 631$ corresponds to the transition from fourth to sixth order polynomial expansion. 

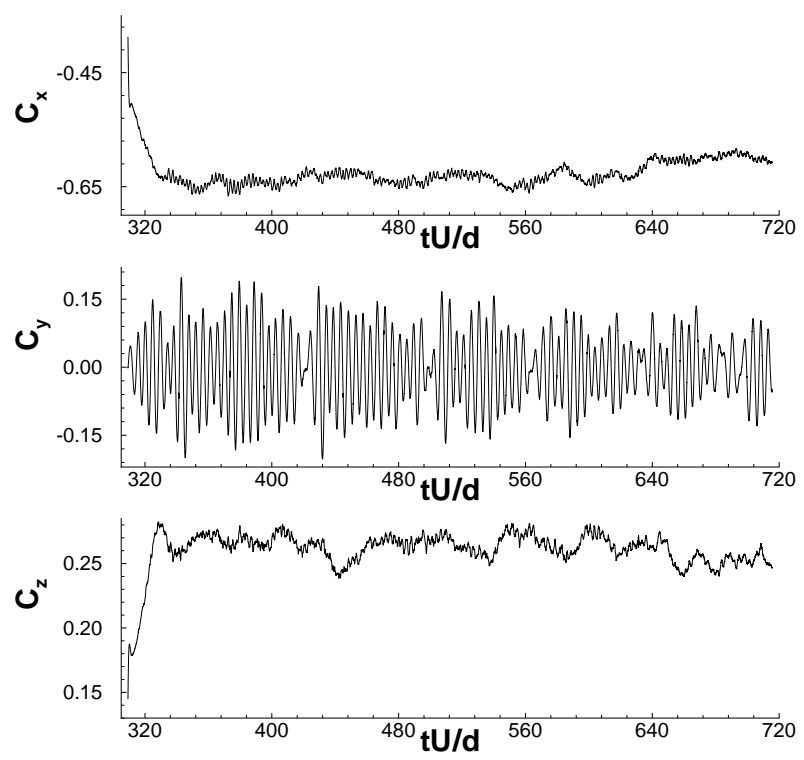

Figure 11. Force history with 4 th and 6 th order polynomials for the first configuration, $R e=500$

The wake topology for this $R e=500$ case, as depicted in figure 12 both in the 3D perspective (a) and in the top view (b), shows vertical rollers and braids inbetween. This sort of flow structures are commonly attributed to Mode A and B transition for a straight cylinder. The flow visualisation for this case, depicted in figure 12(c), is also in good qualitative agreement with the numerical computations.

As depicted in figure 13(a), the pressure contours at slices normal to the cylinder axis now show that the last slice exhibits stronger shedding features than in case 3.1.1. This should be expected, as the local sectional Reynolds number is now higher when compared to that of the same slice along the span for case 3.1.1(a). The sectional forces for $C_{y}$ computed at slices normal to the cylinder axis at different locations of the span for this structure are depicted in figure 13(b). As for case 3.1.1, the top of the cylinder is at $S / d=0$ whereas $S / d=19.6$ corresponds to the end of the quarter turn (S represents the arc length measured from the top). An arc length of $S / d=5$ corresponds to a section inclined approximately $23^{\circ}$ from the horizontal and a local Reynolds number of 345 whereas an arc length of $S / d=15$ corresponds to a section inclined $69^{\circ}$ from the horizontal and a local Reynolds number of roughly 180 . Unlike case 3.1.1, this sectional local Reynolds number is now higher 


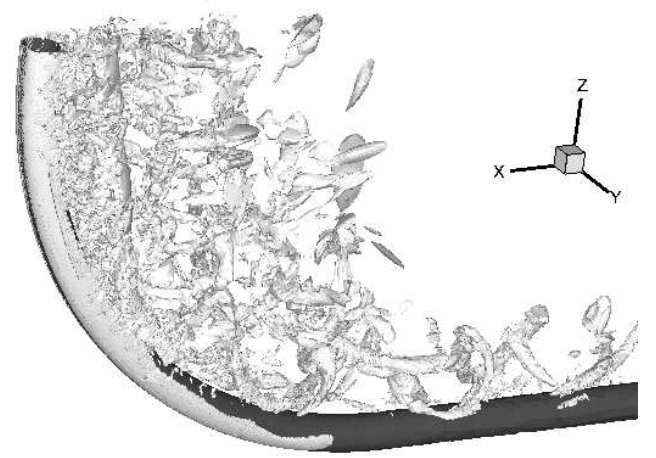

(a)
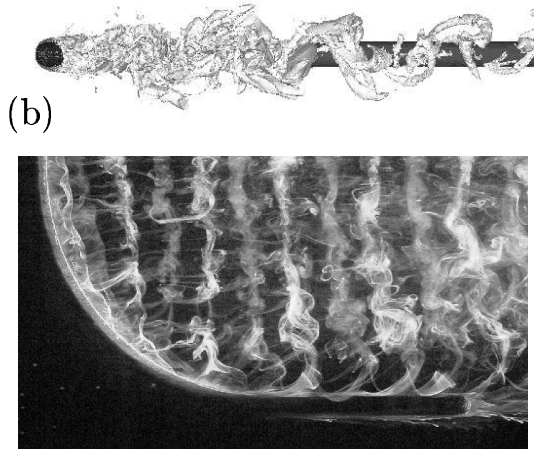

(c)

Figure 12. Vortex cores in the wake of a quarter turn with an in-line extension in uniform parallel flow, $R e=500, \lambda_{2}=-0.8$

than the critical Reynolds number for the vortex shedding of a circular straight cylinder.

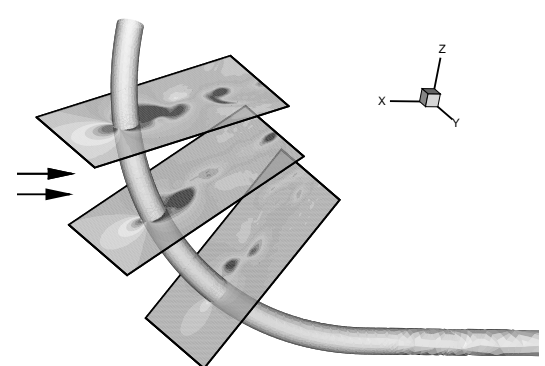

(a)

Figure 13. Pressure contours (a) and $C_{y}$ sectional forces (b) for a quarter turn with an in-line extension in uniform parallel flow, $R e=500$

\subsubsection{Second configuration}

The flow past the configuration studied in section 3.1.2 has also been investigated at $R e=500$. Once again the computation was started originally from the flow field of case 3.1.1 at a Reynolds number of 100 using a fourth order polynomial expansion. 
Preliminary results indicate that similar flow features are observed for this case as with $R e=100$ (steady forces, variation of wake width along the span). The wake topology for the $R e=100$ case is shown in figure 14(a) which illustrates just the shear layers separating from the body but not interacting.

The flow visualisation for the $R e=500$ case is depicted in figure 14(b). Note that because the position of the camera in the experiments was lower than that of the water free surface, a reflection was produced, thus the photograph includes in the top part the mirror image of the flow visualisation in the water surface. Figure 14(b) illustrates that the vortex shedding features are again absent (even though a slight unsteadiness is present at the top). The preliminary computational results suggest that the $R e=500$ case is flow-structurally quite similar to the $R e=100$ case.

(a)

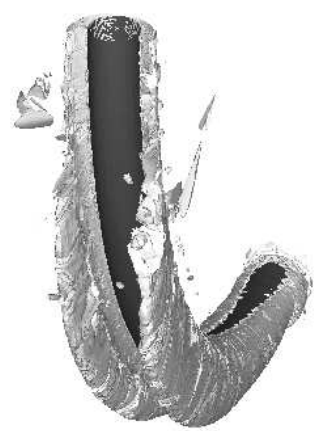

(b)

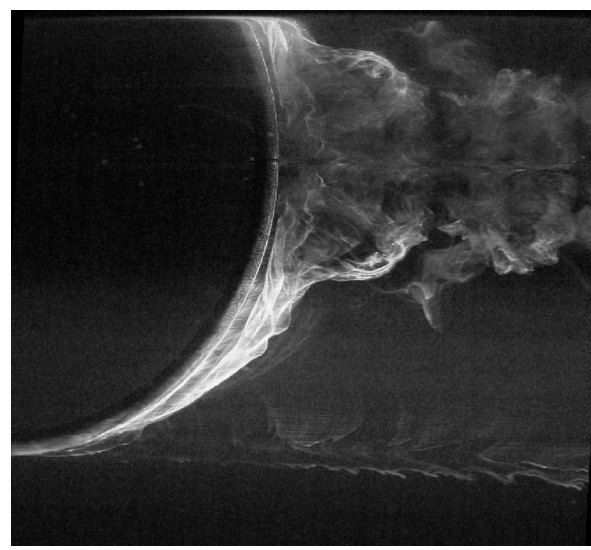

Figure 14. (a) Wake topology for a reverse quarter turn in uniform parallel flow, $R e=100, \lambda_{2}=-0.1$; $\quad$ (b) Flow visualisation for a reverse quarter turn in uniform flow parallel to the plane of curvature, $R e=500$

\section{Conclusions}

In this paper we have presented a computational study of the vortex shedding features of curved cylinders at Reynolds numbers of 100 and 500. The wake topology past curved cylinders at these Reynolds numbers has been investigated and the sectional forces along the axis of the curved cylinder have been presented. The computational results have been confirmed by experiments undertaken in the towing tank facility of the Department of Aeronautics of Imperial College [6].

The two configurations of curved cylinders where the incoming flow is parallel to the plane of curvature have been discussed. For the first 
configuration depicted in Figure 1, which tends to deflect the flow away from the curved cylinder, three-dimensional periodic vortex shedding at $R e=100$ was found to occur towards the top of the body where the cylinder becomes normal to the flow. The variation of the local Reynolds number along the length of the curved cylinder has been discussed in terms of the onset of vortex shedding for a straight twodimensional cylinder. At $R e=500$, braids were observed connecting the vertical rollers, implying the presence of streamwise vorticity in the region connecting the spanwise rollers. These features are commonly associated with transition to three-dimensionality when considering the flow past a straight cylinder. For the configuration depicted in Figure 9 , where flow is deflected into the curved cylinder, however, a steady wake was observed with a variation of the wake width along the span of the cylinder at $R e=100$. Similar features have also been observed both experimentally and computationally for this case at $R e=500$. All the computations were in good qualitative agreement with the flow visualisations from the experiments.

\section{Acknowledgements}

The authors would like to thank C.J. Hogan and J.C. Owen for all their help with the experimental part of this work. Computational resources were provided by the Imperial College Parallel Computing facilities (Fujitsu AP3000) and the Computer Services for Academic Research (CSAR) at the University of Manchester under the EPSRC grant number GR/R64957.

\section{References}

1. Karniadakis, G. E. and Sherwin, S. J.(1999). Spectral/hp Element Methods for $C F D$. Oxford University Press.

2. Jeong, J. and Hussain, F.(1995). On the identification of a vortex. J. Fluid Mech 285, 69-94.

3. Peiro, J., and Sayma, A. I.(1995). A 3-D unstructured multigrid Navier-Stokes solver. In : Numerical Methods for Fluid Dynamics, V. Eds. K. W. Morton and M. J. Baines, Oxford University Press .

4. Peraire, J., Peiro, J., and Morgan, K. (1993). Multigrid solution of the 3-D compressible Euler equations on unstructured tetrahedral grids. Int. J. Numer. Methods Eng. 36, 1029-1044.

5. Karniadakis, G. E., Israeli, M., and Orszag, S. A. (1991). High-order splitting methods for the incompressible Navier-Stokes equations. J. Comput. Phys 97, 414-443.

6. Owen, J. C. (2001). Passive Control of Vortex Shedding in the Wake of Bluff Bodies. Ph.D. thesis, Imperial College of Science, Technology and Medicine. 
7. Sherwin, S. J. and Karniadakis, G. E. (1996). Tetrahedral hp finite elements: Algorithms and flow solutions. Journal of Computational Physics 124, 14-45.

8. Slaouti, A. and Gerrard, J. H. (1981). An experimental investigation of the end effects on the wake of a circular cylinder towed through water at low Reynolds numbers. J. Fluid Mech 112, 297-314.

9. Darekar, R. M. and Sherwin, S. J. (2001). Flow past a square-section cylinder with a wavy stagnation face. J. Fluid Mech 426, 263-295.

10. Bearman, P. W. and Owen, J. C. (1998). Reduction of bluff-body drag and suppression of vortex shedding by the introduction of wavy separation lines. $J$. Fluid Structures 12, 123-130. 